\title{
A Cumulative-signals-based Method for Time Delay Estimation
}

\author{
Volodymyr Mosorov \\ Institute of Applied Computer Science, Faculty of Electrical, Electronic, Computer and Control Engineering, \\ Lodz University of Technology, Poland, Lodz, str. Stefanowskiego 18/22, 90-924, volodymyr.mosorov@p.lodz.pl
}

\begin{abstract}
An original method for time delay estimation is presented. It is based on changing input signals into cumulative ones, followed by determination of inflection points of cumulative curves, and estimation of time delay as time difference of these points' occurrences. To determine the inflection points, a suitable algorithm is proposed. The preliminary results show that the proposed method is sufficiently efficient, especially in the case of flow measurements based on tomography technique when the cross-correlation function of the signals has no evident peak. This method has no limitations on its application for different types of input signals.
\end{abstract}

Keywords: Cumulative signal, time delay, inflection point, signal processing.

\section{INTRODUCTION}

Estimation of time delays in dynamical systems with noise is a common engineering problem which appears in different areas like control performance monitoring of industrial processes, range estimation in radar, and other applications [1], [7], [ 8]. Nowadays, to estimate time delays between two signals, the correlation-based methods or the cross spectral density methods are widely used in practice [2]-[4].

Although these methods have many advantages, some have serious limitations. In practice, the following factors have a strong influence on finding a transit time. The time window must be determined in such a way that the correlation function calculated from the input signals should contain an evident peak. Similarly, for deformed signals, the phase unwrapping is problematic and leads to a loss in performance due to erroneous estimates by using the cross spectral density methods. However, such deformed signals with different lengths very often appear in many industrial applications. One of such examples is process tomography where time delay estimation is widely used to calculate local flow velocity based on pixel changes of tomographic images captured by two sensor planes [5]. Fig.1.a) shows the typical concentration changes within a chosen pixel for two plane sensors as the function of a frame number, and Fig.1.b) shows a graph of the appropriate correlation function. It is worth to note that the shape of proper signals is typically a rectangular one. As it can be seen, the calculated function has no evident peak, since there is an evident plateau from shift \#4 to \#8, and therefore determination of the transit time can be done incorrectly. These limitations, as the mentioned ambiguous function maximum, seriously restrict the application of crosscorrelation methods for time delay measurement and the range of operating conditions where reliable results must be obtained.

The aim of the presented study is to overcome these limitations by using cumulative signals instead of common ones and determine a time delay as delay time between occurrences of global inflection points of cumulative curves. The proposed method can easily be applied in practice and has no limitations for different types of input signals.

\section{METHOD}

Firstly, it is required to determine a time window $T$, containing appropriate signals that will enable time delay to be estimated. In [6] the example of such a method for choosing suitable time window was presented. The idea is to detect the interval when signals will permanently exceed a given threshold value. In such a way we can choose time interval $T=N \Delta T$, where $\Delta T$ - sampling time and $N$ is the total number of samples.

Next step is to convert input signals $s(n)$ into cumulative ones $s_{c}(n)$ as follows:

$$
s_{c}(n)=\sum_{i=0}^{n} s(i), n=1, \ldots, N
$$

where $N$ determines the time window.

It should be noted that the measured signal values $s(n)$ should be positive only. The appropriate cumulative signals of the signals presented in Fig.1.a) are shown in Fig.3. It is obvious that the cumulative signals are increasing and in the case of pulse shape of the input signals, its shape is similar to the integral sign. It is important to note that the curves of the cumulative signals of a pulse shape have always an inflection point. 
The curves of such cumulative signals can be divided into three-time intervals:

- initial interval of small signal's values which corresponds to the lack of an impulse,

- the growing signal whose beginning and end correspond to the beginning and end of the impulse,

- final interval of constant values - no pulse.

The time of occurrence of the inflection point of such cumulative signal corresponds to the time when the maximum of the impulse or its center in the case of rectangular signals appears. The difference between the time of occurrence of the found inflection points of both cumulative signals determines the time delay between two analyzed signals.

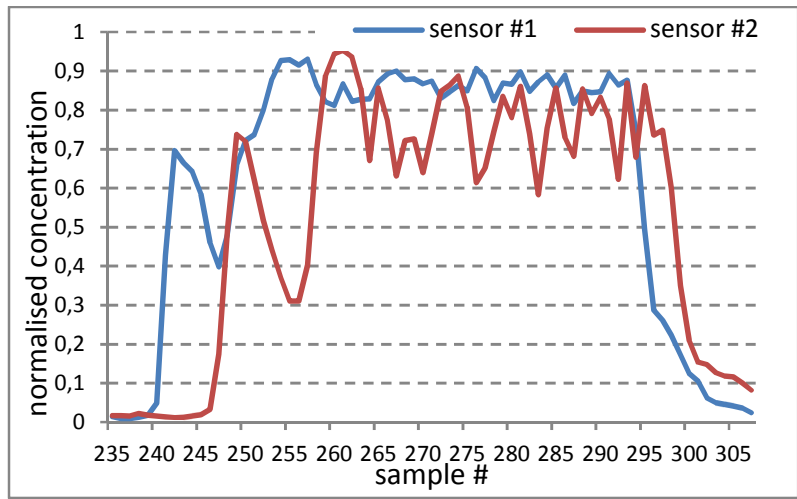

a)

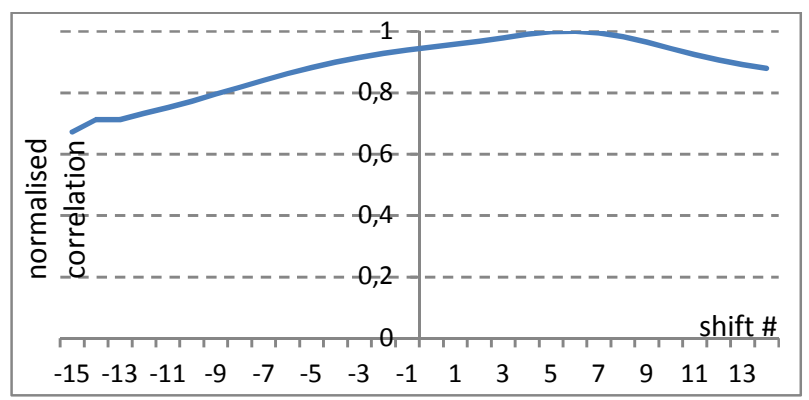

b)

Fig.1. a) Solid concentration changes within chosen pixel from two sensors, b) appropriate calculated correlation function for the time window of 80 samples.

To determine the inflection point of the cumulative signal, the following algorithm was proposed:

Input data: cumulative signal obtained from an impulse digital signal for determined time interval: $s_{c}(n), n=1, \ldots, N$. Output data: The time of occurrence of the inflection point. Algorithm: The inflection point of the cumulative signal is determined as the point of intersection of the signal $s_{c}$ by a straight-line $l$, where the line $l$ is defined on the basis of criterion (1) or (2):

1) $\min \left(S_{u}^{n}-S_{a}^{n}\right), n=1, \ldots, N$, when $S_{u}^{1} \geq S_{a}^{1}$

where $S_{u}^{n},\left(S_{a}^{n}\right)$ is the area under(above) straight line $l$ and above(under) cumulative signal curve $s_{c}$ respectively, and line $l$ passes through points $\left(n, s_{c}(n)\right)$ and $\left(N, s_{c}(N)\right)$
2) $\min \left(S_{a}^{N-n}-S_{u}^{N-n}\right), n=1, \ldots, N$, when $S_{u}^{1}<S_{a}^{1}$ where $S_{a}^{n-n},\left(S_{u}^{N-n}\right)$ is the area above(under) straight line $l$ and above(under) cumulative signal curve $s_{c}$ respectively, and line $l$ passes through points $\left(1, s_{c}(1)\right)$ and $\left(N-n, s_{c}(N-\right.$ n)).

Fig.2. shows an example of determining an inflection point using the proposed algorithm for both cases (1) and (2). We search for a case when areas $S_{a}^{i}$ and $S_{u}^{i}$ determine the straight line $l$ and the cumulative curve $s_{c}$ will be maximally equal.

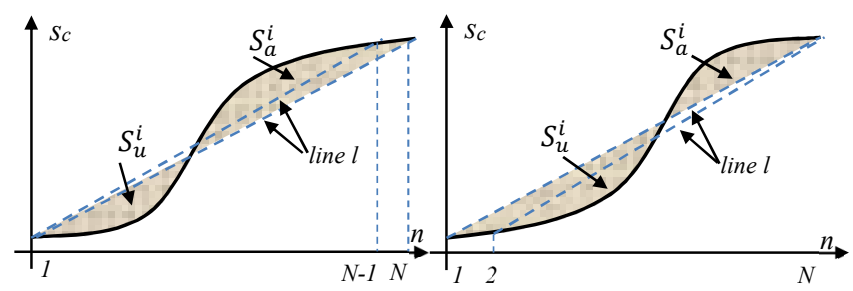

a)

b)

Fig.2. Example of finding the inflection point based on comparison of the areas $S_{u}^{i}$ and $S_{a}^{i}$ determined by cumulative signal $s_{c}$ and straight-line $l$ using criterion: a) \#1 b) \#2.

\section{RESULTS}

Fig.3. shows the found inflection points of the cumulative signals presented in Fig.1.a) using the proposed algorithm. First inflection point occurs at \#270 sample and the second one at $\# 275$, hence time delay between the input signals is equal to 5-time sampling intervals.

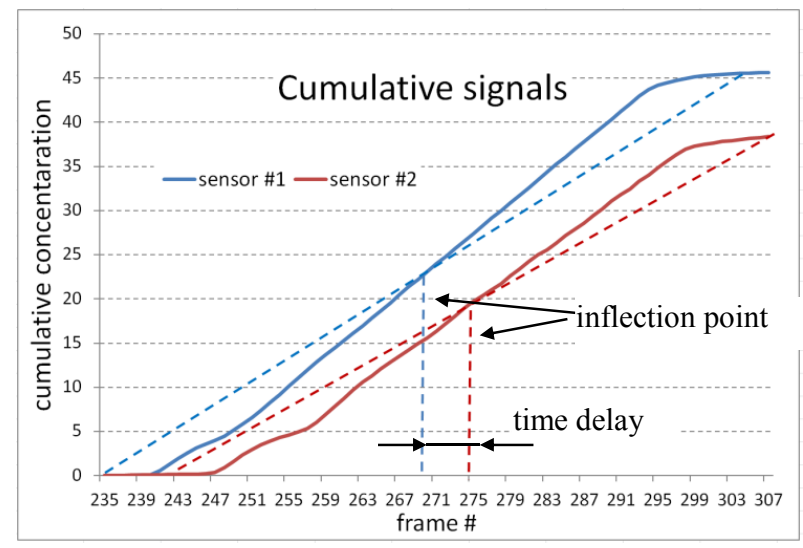

Fig.3. Cumulative signals of signals shown in Fig.1.a). Dashed line represents the best fitted straight line minimizing criterion (1) or (2). Inflection points: \# 270 (sensor \#1) \#275 (sensor \#2).

Efficiency of the proposed method was also validated by simulation. The simulation results were obtained for a rectangular shape of the impulses shifted for 5 time sampling intervals. The signals were noised by additive noise with uniform distribution. Additionally, one of the impulses was shorter than another one by $10 \%$. Example of such noised impulses is shown in Fig.4.a). The appropriate calculated cumulative signals and correlation function are shown in Fig.4.b) and Fig.5., respectively. 


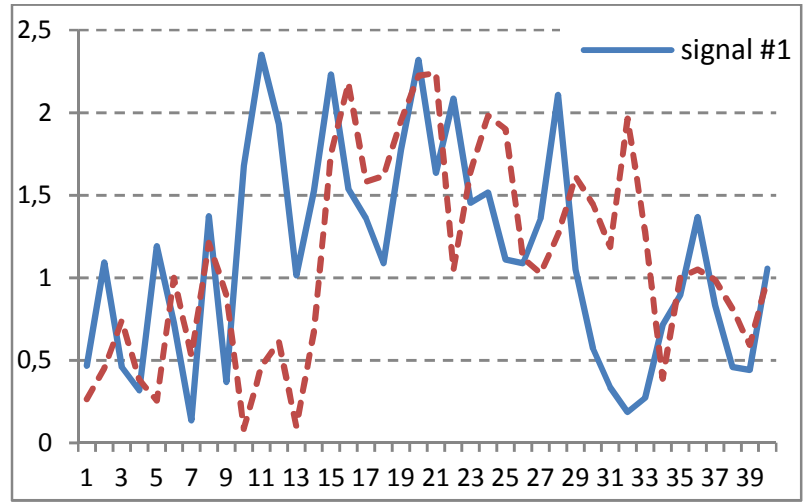

a)

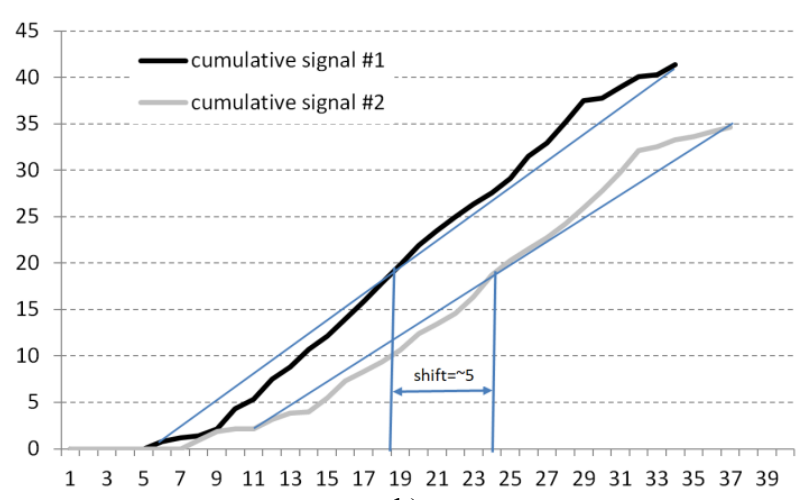

b)

Fig.4. Simulated signals SNR $=8 \mathrm{~dB}$ a) and appropriate calculated cumulative signals $b$ ).

Fig.6. compares the relative errors of shift estimation of two algorithms: classical correlation method and the proposed one. The relative error was calculated as $\frac{1}{N} \sum_{n=1}^{N} \frac{\mid \text { Shift }_{\text {est }}^{n}-\text { Shift }_{\text {true }} \mid}{\text { Shift } t_{\text {true }}} 100 \%$, where Shift $t_{\text {est }}^{n}$ and Shift $t_{\text {true }}$ are the shifts estimated by classical/proposed method in $n$th simulation and true shift value, respectively. Total number of simulations was 100 .

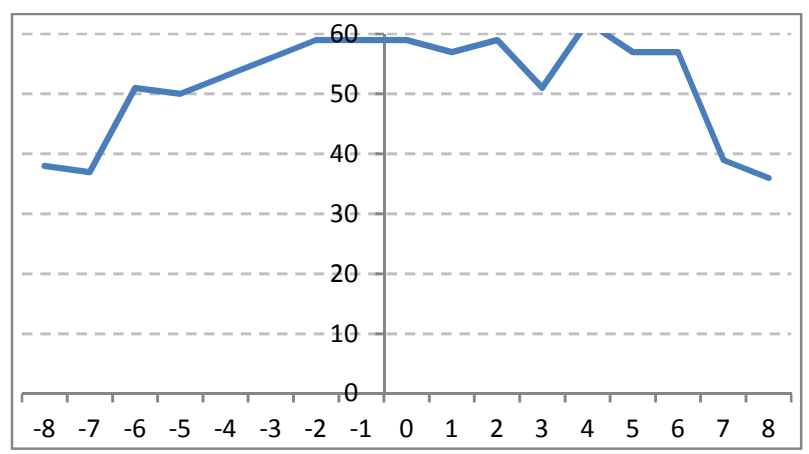

Fig.5. Correlation function of signals shown in Fig.4. for the time window of 35 samples.

As it can be seen in Fig.6., relative error of the time delay estimation for the case of the low noised signals is the same for both methods. However, when SNR falls down $(\mathrm{SNR}<8 \mathrm{~dB})$, the proposed method allows to estimate a time delay more accurately.

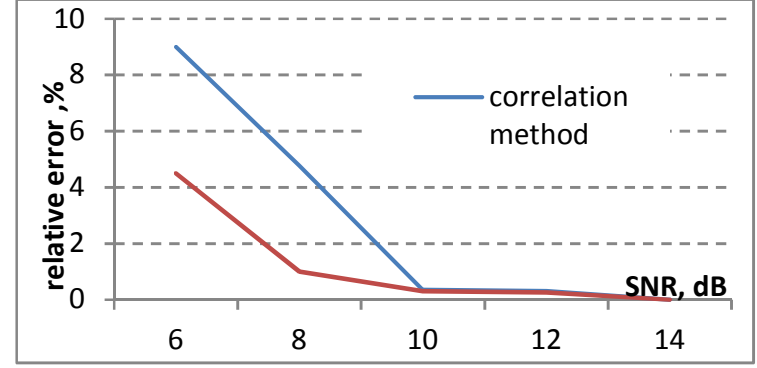

Fig.6. Relative error of time delay estimation by two methods.

\section{CONCLUSION}

A more accurate method for estimating time delay of highly noised signals is presented here. It is based on determination of time delay as a difference between inflection points of cumulative signals calculated from input signals. Contrary to the classical cross-correlation methods, this method is more robust on noise, changes of signal shapes and its length. Additionally, the algorithm procedure is clearer and allows avoiding ambiguity. The preliminary results show that it can be used for any signal in a wide range of different applications.

\section{REFERENCES}

[1] Bendat, J.S., Piersol, A.G. (2010). Random Data Analysis and Measurement Procedures, Fourth Edition. Wiley.

[2] Jacovitti, G., Scarano, G. (1993). Discrete time technique for time delay estimation. IEEE Transactions on Signal Processing, 41 (2), 525-533.

[3] Hanus, R., Kowalczyk, A., Szlachta, A., Chorzępa, R. (2018). Application of conditional averaging to time delay estimation of random signals. Measurement Science Review, 18 (4), 130-137.

[4] Hanus, R., Zych, M., Petryka, L., Świsulski, D. (2014). Time delay estimation in two-phase flow investigation using the $\gamma$-ray attenuation technique. Mathematical Problems in Engineering, 2014, 475735.

[5] Mosorov, V. (2006). Phase spectrum method for time delay estimation using twin plane electrical capacitance tomography. Electronics Letters, 42 (11), 630-632.

[6] Mosorov, V. (2008). Flow pattern tracing for mass flow rate measurement in pneumatic conveying using twin plane electrical capacitance tomography. Particle \& Particle Systems Characterization, 25 (3), 259-265.

[7] Roshani, G.H., Nazemi, E. Roshani, M.M. (2017). Usage of two transmitted detectors with optimized orientation in order to three phase flow metering. Measurement, 100 (1), 122-130.

[8] Zych, M., Hanus, R., Wilk, B., Petryka, L., Świsulski, D. (2018). Comparison of noise reduction methods in radiometric correlation measurements of two-phase liquid-gas flows. Measurement, 129, 288-295.

Received October 25, 2018 Accepted March 29, 2019 